\title{
Time-resolved resonant soft $x$-ray diffraction with free-electron lasers: Femtosecond dynamics across the Verwey transition in magnetite
}

\author{
N. Pontius, ${ }^{1, a)}$ T. Kachel, ${ }^{1}$ C. Schüßler-Langeheine, ${ }^{1,2}$ W. F. Schlotter, ${ }^{3,4}$ M. Beye, ${ }^{1,3}$ \\ F. Sorgenfrei, ${ }^{3}$ C. F. Chang, ${ }^{2}$ A. Föhlisch, ${ }^{1,3,5}$ W. Wurth, ${ }^{3}$ P. Metcalf, ${ }^{6}$ I. Leonov, ${ }^{7}$ \\ A. Yaresko, ${ }^{8}$ N. Stojanovic, ${ }^{9}$ M. Berglund,,${ }^{1,3,5}$ N. Guerassimova, ${ }^{9}$ S. Düsterer, ${ }^{9}$ \\ H. Redlin, ${ }^{9}$ and H. A. Dürr ${ }^{4,10}$ \\ ${ }^{1}$ Institute for Methods and Instrumentation in Synchrotron Radiation Research, Helmholtz-Zentrum Berlin \\ für Materialien und Energie GmbH, Albert-Einstein-Str. 15, 12489 Berlin, Germany \\ ${ }^{2}$ II. Physikalisches Institut, Universität zu Köln, Zülpicher Straße 77, 50937 Köln, Germany \\ ${ }^{3}$ Department of Physics and Center for Free-Electron Laser Science, Universität Hamburg, \\ Luruper Chaussee 149, 22761 Hamburg, Germany \\ ${ }_{5}^{4}$ SLAC National Accelerator Laboratory, Menlo Park, California 94025, USA \\ ${ }^{5}$ Institut für Physik und Astronomie, Universität Potsdam, Karl-Liebknecht-Straße 24-25, \\ 14476 Potsdam, Germany \\ ${ }^{6}$ School of Materials Engineering, Purdue University, West Lafayette, Indiana 47907, USA \\ ${ }^{7}$ Theoretical Physics III, Institute of Physics, University of Augsburg, Augsburg 86135, Germany \\ ${ }^{8}$ Max Planck Institute for Solid State Research, Heisenbergstraße 1, 70569 Stuttgart, Germany \\ ${ }^{9}$ Deutsches Elektronen-Synchrotron DESY, Notkestraße 85, 22607 Hamburg, Germany \\ ${ }^{10}$ Department for Magnetic Dynamics, Helmholtz-Zentrum Berlin für Materialien und Energie GmbH, \\ Albert-Einstein-Str. 15, 12489 Berlin, Germany
}

(Received 21 December 2010; accepted 17 March 2011; published online 5 May 2011)

\begin{abstract}
Resonant soft x-ray diffraction (RSXD) with femtosecond (fs) time resolution is a powerful tool for disentangling the interplay between different degrees of freedom in strongly correlated electron materials. It allows addressing the coupling of particular degrees of freedom upon an external selective perturbation, e.g., by an optical or infrared laser pulse. Here, we report a time-resolved RSXD experiment from the prototypical correlated electron material magnetite using soft $\mathrm{x}$-ray pulses from the free-electron laser FLASH in Hamburg. We observe ultrafast melting of the charge-orbital order leading to the formation of a transient phase, which has not been observed in equilibrium. (C) 2011 American Institute of Physics. [doi:10.1063/1.3584855]
\end{abstract}

Resonant x-ray diffraction, i.e., an x-ray diffraction experiment with the photon energy tuned into resonance with a dipole transition, combines the high spectroscopic sensitivity of x-ray absorption spectroscopy with momentum resolution. Thus it provides not only information on structural aspects of a material but also on its electronic properties. Particularly interesting in this regard are the strong dipole allowed excitations into transition-metal $3 d$, oxygen $2 p$, and lanthanide $4 f$-states, which all occur in the soft x-ray range between 400 and $1600 \mathrm{eV}$. Within the momentum space that can be probed with resonant soft $\mathrm{x}$-ray diffraction are antiferromagnetic reflections from natural or artificial magnetic structures and superstructure reflections caused by periodic modulations of the electronic state as they occur in a large class of correlated electron systems. ${ }^{1}$

A prototype material from the latter is magnetite $\left(\mathrm{Fe}_{3} \mathrm{O}_{4}\right)$. Already in the late 1930 s Verwey discovered that upon lowering of the temperature below $\mathrm{T}_{\mathrm{V}}=123 \mathrm{~K}$ (the Verwey temperature), magnetite undergoes a first-order phase transition leading to a conductivity decrease by two orders of magnitude. ${ }^{2}$ This Verwey transition involves a change from a cubic inverse spinel high-temperature lattice structure to a complex monoclinic low-temperature phase. ${ }^{3}$ Verwey proposed as the mechanism behind the drop in electrical conductivity a freezing of the charge fluctuations between octahedrally coordinated (B-site) $\mathrm{Fe}^{2+}$ and $\mathrm{Fe}^{3+}$ ions

${ }^{a)}$ Electronic mail: niko.pontius@helmholtz-berlin.de. into a charge ordered structure. Calculations further predicted orbital order, i.e., a spatial modulation of the orbital occupation, for the low-temperature phase..$^{4-8}$ Both charge and orbital order have been observed experimentally, ${ }^{3,6,9-11}$ even though no full consensus exists over all aspects of the order.

One of the superstructure peaks characteristic of the lowtemperature phase is $(0,0,1 / 2)$ (notation refers to the cubic room temperature unit cell with $a=8.39 \AA$ ). The inset to Fig. 1 shows a scan through this peak along the [001] (L) direction in reciprocal space. In the main panel of Fig. 1 the photon energy dependence of the $(0,0,1 / 2)$-peak intensity (red) at the oxygen $1 s \rightarrow 2 p$ (K) resonance is compared to the $\mathrm{x}$-ray absorption spectrum (black) from the same sample. Since $\mathrm{x}$-ray absorption probes the whole unoccupied $p$-derived conduction-band density of states, the energy position of the $(0,0,1 / 2)$ resonance clearly shows that this peak reflects a spatial modulation of states at the bottom of the conduction band. Local-density approximation (LDA+U) calculations reveal that these states are sensitive to charge/ orbital order via $\mathrm{O} 2 p-\mathrm{Fe} 3 d$ hybridization. ${ }^{7,11}$ It is these states that also form the energy gap ${ }^{12,13}$ leading to the insulating nature of magnetite below $\mathrm{T}_{\mathrm{V}}$. Hence, the $(0,0,1 / 2)$ diffraction peak provides us with direct information about the electronic order as well as the energy position of the involved electronic levels. By probing this signal time resolved, we can study the melting of charge/orbital order as well as a change in the band structure as a response to an electronic excitation induced with an infrared laser pulse. 


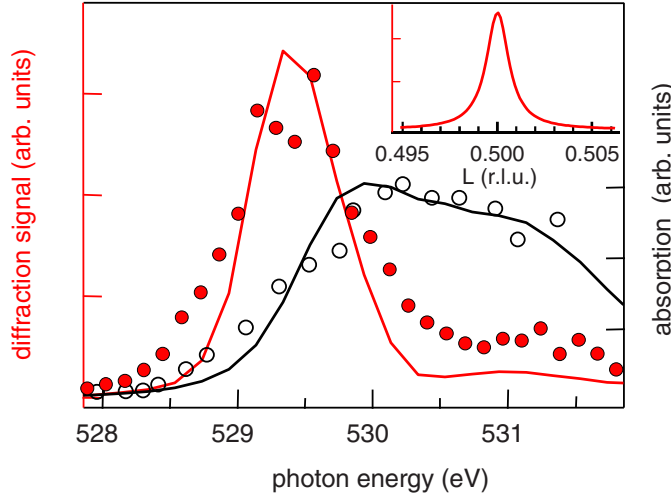

FIG. 1. (Color online) Oxygen $K$-edge $x$-ray absorption spectra and $(0,0,1 / 2)$ RSXD of magnetite $(\mathrm{T}=90 \mathrm{~K})$. The open (filled) symbols represent the fluorescence yield (RSXD signal) recorded at FLASH, the corresponding lines represents the total electron yield (RSXD signal) recorded at BESSY. The inset shows a scan along [001] (L) through the $(0,0,1 / 2)$ RSXD peak. The FWHM value of 0.0015 r.l.u. (reciprocal lattice units) of the $(0,0,1 / 2)$ diffraction peak is determined by the $\mathrm{x}$-ray penetration depth

The time-resolved resonant soft $\mathrm{x}$-ray diffraction (RSXD) measurements were carried out at the PG2 beamline $^{14,15}$ of the free-electron laser facility FLASH at DESY (Hamburg), using monochromatized x-rays of the third undulator harmonic. The monochromator was set to $\sim 1 \mathrm{eV}$ photon energy resolution $(200 \mathrm{l} / \mathrm{mm}$ grating, $\mathrm{c}_{\mathrm{ff}}$-value $\left.=1.18\right)$ matching the spectral width of the resonant diffraction peak (Fig. 1). Photon energy scans across the Oxygen $1 s \rightarrow 2 p$ resonance were possible within the FLASH $\sim 1 \%$ photon energy bandwidth (i.e., $\sim 5 \mathrm{eV}$ ). ${ }^{16}$ FLASH was operated at a pulse train repetition rate of $5 \mathrm{~Hz}$ with each train (macrobunch) containing 30 microbunches with an internal repetition rate of $500 \mathrm{kHz}$. The FLASH pulses were typically 40 fs long. ${ }^{17}$ When compared to the $500 \mathrm{MHz}$ repetition rate operation of synchrotrons this resulted in similar average photon flux even in the third harmonic of the freeelectron laser. In Fig. 1 we compare the spectra recorded at FLASH (symbols) with those recorded at a third generation synchrotron-radiation facility BESSY II in Berlin (lines). Despite the intrinsic intensity fluctuations of a SASE-FEL, ${ }^{16}$ spectra with comparable signal-to-noise ratio are obtained at both sources.

A femtosecond (fs) pump-laser ${ }^{18}(\mathrm{~h} \nu=1.5 \mathrm{eV}, 130 \mathrm{fs}$ pulse duration) was synchronized to every other $\mathrm{x}$-ray pulse $(250 \mathrm{kHz})$ allowing for proper normalization by measuring the sample alternating between the ground and the excited state. Zero time delay between infrared pump and x-ray probe pulses was narrowed down to a $\sim 20$ ps window using an in situ high-bandwidth copper photocathode. The pump laser focus on the sample had a $\sim 250 \mu \mathrm{m}$ full width at half maximum (FWHM) diameter providing pump-fluences up to $10 \mathrm{~mJ} / \mathrm{cm}^{2}$. High-quality synthetic magnetite single crystals were cleaved in situ in UHV. Measurements were performed at $90 \mathrm{~K}$ sample temperature. The diffracted $\mathrm{x}$-rays were detected by a $\sim 3 \mathrm{~mm}$ diameter ( $5^{\circ}$ acceptance angle) fast avalanche photodiode (Silicon APD, Laser Components) integrating over the whole diffraction peak. The APD was screened from the pump-laser photons by an optically tight $500 \mathrm{~nm}$ thick $\mathrm{Al}$ foil. The amplified fast diode signal was directly recorded with a $2 \mathrm{GHz}$ sampling rate resolving each individual microbunch contribution. The varying temporal jitter between FLASH and fs pump-laser pulses was separately measured for each macro bunch individually using

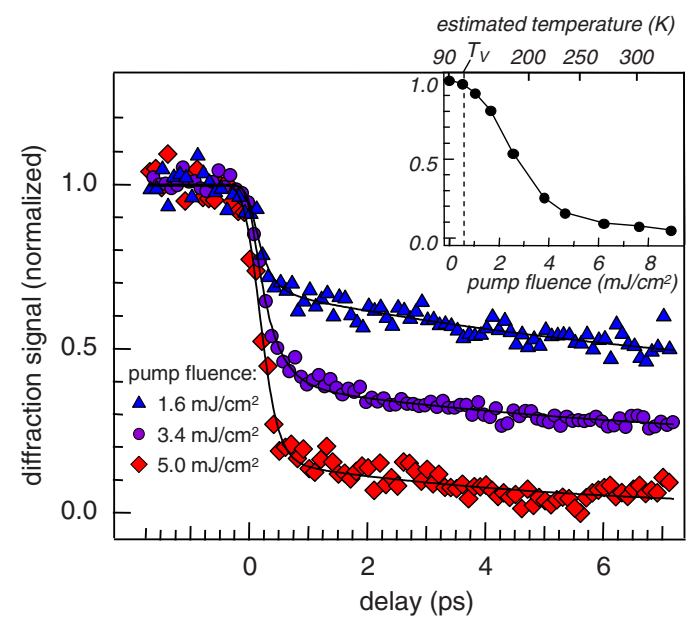

FIG. 2. (Color online) Time-dependent oxygen $K$-edge $(0,0,1 / 2)$ RSXD signal vs. laser pump and x-ray probe time-delay for three different fs-laser pulse fluences (symbols). The lines represent double exponential fits. The inset shows the fluence dependence of the $(0,0,1 / 2)$ RSXD signal measured at 200 ps time delay measured at BESSY II.

electro-optical sampling (EOS). ${ }^{19}$ Reference 20 demonstrates that by using EOS for jitter correction a total temporal resolution of $210 \mathrm{fs}$ can be achieved.

In order to study the response of the system to an infrared fs-laser pulse at time $t_{0}$, we monitored the $(0,0,1 / 2)$ diffraction peak intensity at a second time $t_{0}+\Delta t$ using a soft $\mathrm{x}$-ray pulse from FLASH. Figure 2 shows the $(0,0,1 / 2)$ RSXD peak intensity at $529.4 \mathrm{eV}$ photon energy (peak maximum) as a function of time delay, $\Delta \mathrm{t}$, for three pump laser fluences 1.6, 3.4, and $5.0 \mathrm{~mJ} / \mathrm{cm}^{2}$ (sample surface). The peak intensity drops by more than $90 \%$ within the first ps for the highest pump fluence, while the two lower pump fluences lead to a reduction to $\sim 40 \%$ and $\sim 70 \%$, respectively, of the initial value. To determine the time constants from the delay scans in Fig. 2, we fitted the data with a double exponential decay convoluted by a Gaussian taking the FLASH temporal resolution into account, which is mainly determined by the arrival time jitter between the two pulses. ${ }^{20}$ This procedure yields that for all laser fluences the initial fast decay is essentially resolution limited which implies a time constant $<270$ fs. In all cases, a subsequent slower decay with smaller amplitudes of $\sim 20 \%$ and $\sim 10 \%$ follows, the timescale of which we estimate to several ten ps indicating its relationship with a lattice rearrangement. The inset to Fig. 2 shows the intensity that remains 200 ps after laser excitation as a function of pump fluence measured at BESSY II.

To observe residual $(0,0,1 / 2)$-peak intensity $200 \mathrm{ps}$ after the pump pulse is remarkable because the thermalization of the energy deposited by the pump pulse should be completed within a few ps. The sample volume-averaged temperature in the probed sample volume ${ }^{21}$ resulting from this thermalization is shown as the scale on top of the inset of Fig. 2.2,23 Except for the weakest, all pump pulses heat the probed sample volume well above $T_{V}=123 \mathrm{~K}$. The residual peak intensity observed picoseconds after the pump pulse hence comes from a non-equilibrium transient phase.

In order to characterize the transient phase we make use of the spectroscopic sensitivity of our method. Figure 3 shows a false-color map of the $(0,0,1 / 2)$ peak intensity as a function of delay time and photon energy for a pump fluence of $4 \mathrm{~mJ} / \mathrm{cm}^{2}$. The data were recorded by scanning the monochromator and the delay stage for the pump pulses si- 


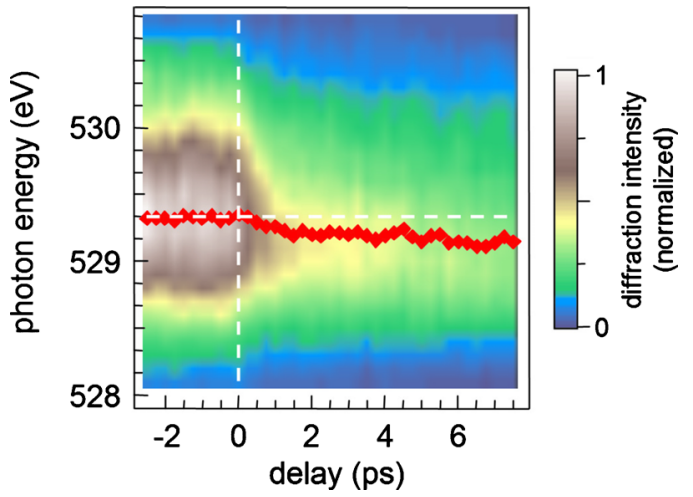

FIG. 3. (Color online) Oxygen $K$-edge $(0,0,1 / 2)$ RSXD intensity vs. photon energy and time delay. The diamonds mark the resonance peak maxima on the energy scale as determined by individual fits of Gaussian peak profiles to the raw data. The fs laser pump fluence was set to $4 \mathrm{~mJ} / \mathrm{cm}^{2}$.

multaneously back and forth with sizable different speeds such that the points in the map were recorded in an uncorrelated sequence. This approach averages efficiently over possible long-term drifts in the experimental setup. The temporal resolution for this protracted measurement was $750 \mathrm{fs}$. We find that the transition from the low-temperature to the transient phase is accompanied by a $0.18 \pm 0.05 \mathrm{eV}$ energy shift in the resonance peak to lower photon energies.

The $180 \mathrm{meV}$ size of the energy shift is remarkably large compared to the $\sim 40 \mathrm{meV}$ shift observed in $\mathrm{x}$-ray absorption spectroscopy experiments at the Verwey transition. ${ }^{24}$ In fact the measured shift in the resonance amounts to about the full size of the band gap of $230 \mathrm{meV}$ in the low-temperature phase. ${ }^{7}$ The huge shift in the resonance energy that we observe strongly supports our arguments that a so far unknown transient phase is formed in the first several picoseconds after the pump pulse and we conclude that this transient phase is characterized by the existence of partial charge/orbital order in the presence of an almost closed band gap.

In conclusion we demonstrated how a fs-infrared-laserpump x-ray-free-electron-laser-probe resonant soft x-ray diffraction experiment provides insights into the dynamics of phase transitions and further allows to prepare and to characterize so far unknown transient phases of matter. This approach opens the door for further studies such as directly detecting spin, charge and orbital excitations in real time..$^{25}$ Improvements in beamline efficiency at FLASH and $\mathrm{x}$-ray free-electron lasers that are presently coming online will widen the range of systems that can be studied considerably.

We thank D. Khomskii, S. Bonhommeau, and K. Holldack for helpful discussions. This research was supported by the German Bundesministerium für Bildung und Forschung in the framework of FSP 301 (Priority program FLASH), by the DFG through SFB 608 and SFB 484, by the European Union and by the U.S. Department of Energy, Office of Basic Energy Sciences under Contract No. DE-AC0276SF00515. We are indebt to the scientific and technical team at FLASH, in particular, the machine operators and run coordinators (especially B. Faatz and R. Treusch), being the foundation of the successful operation and delivery of the SASE-FEL beam.

${ }^{1}$ M. Imada, A. Fujimori, and Y. Tokura, Rev. Mod. Phys. 70, 1039 (1998).

${ }^{2}$ E. J. W. Verwey, Nature (London) 144, 327 (1939).

${ }^{3}$ J. P. Wright, J. P. Attfield, and P. G. Radaelli, Phys. Rev. Lett. 87, 266401 (2001); Phys. Rev. B 66, 214422 (2002).

${ }^{4}$ P. Piekarz, K. Parlinski, and A. M. Oles, Phys. Rev. Lett. 97, 156402 (2006).

${ }^{5}$ F. Walz, J. Phys.: Condens. Matter 14, R285 (2002).

${ }^{6}$ H.-T. Jeng, G. Y. Guo, and D. J. Huang, Phys. Rev. Lett. 93, 156403 (2004).

${ }^{7}$ I. Leonov, A. N. Yaresko, V. N. Antonov, M. A. Korotin, and V. I. Anisimov, Phys. Rev. Lett. 93, 146404 (2004).

${ }^{8}$ I. Leonov, A. N. Yaresko, V. N. Antonov, and V. I. Anisimov, Phys. Rev. B 74, 165117 (2006).

${ }^{9}$ J. Schlappa, C. Schüßler-Langeheine, C. F. Chang, H. Ott, A. Tanaka, Z. Hu, M. W. Haverkort, E. Schierle, E. Weschke, G. Kaindl, and L. H. Tjeng, Phys. Rev. Lett. 100, 026406 (2008).

${ }^{10}$ E. Nazarenko, J. E. Lorenzo, Y. Joly, J. L. Hodeau, D. Mannix, and C. Marin, Phys. Rev. Lett. 97, 056403 (2006).

${ }^{11}$ D. J. Huang, H. Lin, J. Okamoto, K. S. Chao, H. Jeng, G. Y. Guo, C. Hsu, C. Huang, D. C. Ling, W. B. Wu, C. S. Yang, and C. T. Chen, Phys. Rev. Lett. 96, 096401 (2006).

${ }^{12}$ S. K. Park, T. Ishikawa, and Y. Tokura, Phys. Rev. B 58, 3717 (1998).

${ }^{13}$ J. H. Park, L. H. Tjeng, J. W. Allen, P. Metcalf, and C. T. Chen, Phys. Rev. B 55, 12813 (1997).

${ }^{14}$ M. Martins, M. Wellhöfer, J. T. Hoeft, W. Wurth, J. Feldhaus, and R. Follath, Rev. Sci. Instrum. 77, 115108 (2006).

${ }^{15}$ M. Wellhöfer, M. Martins, W. Wurth, A. A. Sorokin, and M. Richter, J. Opt. A, Pure Appl. Opt. 9, 749 (2007).

${ }^{16}$ W. Ackermann, G. Asova , V. Ayvazyan et al., Nat. Photonics 1, 336 (2007).

${ }^{17}$ U. Frühling, M. Wieland, M. Gensch, T. Gebert, B. Schütte, M. Krikunova, R. Kalms, F. Budzyn, O. Grimm, J. Rossbach, E. Plönjes, and M. Drescher, Nat. Photonics 3, 523 (2009).

${ }^{18}$ H. Redlin, A. Al-Shemmary, A. Azima, N. Stojanovic, F. Tavella, I. Will, and S. Düsterer, Nucl. Instrum. Methods Phys. Res. A 635, S88 (2011)

${ }^{19}$ A. L. Cavalieri, D. M. Fritz, S. H. Lee, P. H. Bucksbaum, D. A. Reis, J. Rudati, D. M. Mills, P. H. Fuoss, G. B. Stephenson, C. C. Kao, D. P. Siddons, D. P. Lowney, A. G. MacPhee, D. Weinstein, R. W. Falcone, R. Pahl, J. Als-Nielsen, C. Blome, S. Düsterer, R. Ischebeck, H. Schlarb, H. Schulte-Schrepping, T. Tschentscher, J. Schneider, O. Hignette, F. Sette, K. Sokolowski-Tinten, H. N. Chapman, R. W. Lee, T. N. Hansen, O. Synnergren, J. Larsson, S. Techert, J. Sheppard, J. S. Wark, M. Bergh, C. Caleman, G. Huldt, D. van der Spoel, N. Timneanu, J. Hajdu, R. A. Akre, E. Bong, P. Emma, P. Krejcik, J. Arthur, S. Brennan, K. J. Gaffney, A. M. Lindenberg, K. Luening, and J. B. Hastings, Phys. Rev. Lett. 94, 114801 (2005).

${ }^{20}$ A. Azima, S. Düsterer, P. Radcliffe, H. Redlin, N. Stojanovic, W. Li, H. Schlarb, J. Feldhaus, D. Cubaynes, M. Meyer, J. Dardis, P. Hayden, P. Hough, V. Richardson, E. T. Kennedy, and J. T. Costello, Appl. Phys. Lett. 94, 144102 (2009).

${ }^{21}$ Estimation of the sample temperature considers the scattering geometry for derivation of the effective surface laser fluence and penetration depth (the latter is $105 \mathrm{~nm}$ as deduced from Ref. 22), a surface reflectivity of 6\% derived for p-polarized light at $800 \mathrm{~nm}$, the temperature dependent heat capacity of magnetite from Ref. 23 , and averages the temperature over a volume given by the x-ray penetration depth of $46 \mathrm{~nm}$.

${ }^{22}$ A. Schlegel, S. F. Alvarado, and P. Wachter, J. Phys. C: Solid State Phys. 12, 1157 (1979).

${ }^{23}$ J. P. Shepherd, J. W. Koenitzer, R. Aragon, and J. M. Sandberg, Phys. Rev. B 31, 1107 (1985).

${ }^{24}$ E. Goering, S. Gold, M. Lafkioti, G. Schütz, and V. A. M. Brabers, Phys. Rev. B 72, 033112 (2005).

${ }^{25}$ D. Polli, M. Rini, S. Wall, R. W. Schoenlein, Y. Tomioka, Y. Tokura, G. Cerullo, and A. Cavalleri, Nature Mater. 6, 643 (2007). 